\title{
Availability Formulations for Segment Protection
}

\author{
Massimo Tornatore, Matteo Carcagní, and Achille Pattavina
}

\begin{abstract}
Segment Protection (SP) is an efficient scheme for protection in WDM optical networks. This letter provides algebraic formulations to evaluate SP availability both in the dedicated and shared backup case. The availability models are applied to numerical examples and significant relations between SP availability and relevant connection parameters are identified.
\end{abstract}

Index Terms-Optical network, availability, shared-segment protection.

\section{INTRODUCTION}

$\mathbf{T}$ HANKS to the wavelength-division-multiplexing (WDM) technology, today's optical networks provide a transport infrastructure with very high capacity. This huge capacity requires efficient survivability mechanisms in order to avoid that the failure of a network element (typically a link or a node) can cause a large amount of data loss. Recently, new techniques have been proposed to efficiently deal with this problem in mesh networks. Among them, Segment Protection (SP) is a promising candidate for protection in WDM/MPLS networks because of its desirable resource efficiency, and for its ability to limit the signalling delay for recovery.

Before the activation of an optical connection over a WDM network, an Optical-Service Level Agreement (O-SLA), that specifies the Quality-of-Service requirements to be guaranteed by the optical circuit, has to be contracted between the client and the optical operator. Among the various parameter that form an O-SLA, we focus our attention on the connection availability, which is widely recognized as one of the key parameters to set the class of service for optical circuits. In this work we intend to provide a rigorous methodology to evaluate the availability level provided by SP-protected connections, in order to verify if the availability targets of the SP-protected connections are met.

\section{Shared Segment Protection}

Various forms of segment protection have been proposed in the technical literature [1]-[4]. The common idea of these approaches is to divide a working path (WP) into several working segments (WSs) and to protect each WS with a node/linkdisjoint backup segment (BS). When a failure occurs, only

Paper approved by K. Kitayama, the Editor for Photonic Networks and Fiber Optic Wireless of the IEEE Communications Society. Manuscript received July 31, 2008; revised September 8, 2009.

The authors are with the Department of Electronics and Information, Politecnico di Milano, Via Ponzio 34-35, 20121 Milan, Italy (e-mail: \{tornator, pattavina\}@elet.polimi.it, matteo.carcagni@alcatel-lucent.com).

The work described in this paper was carried out with the support of the BONE-project ("Building the Future Optical Network in Europe"), a Network of Excellence funded by the European Commission through the 7th ICTFramework Programme.

Digital Object Identifier 10.1109/TCOMM.2010.04.080103

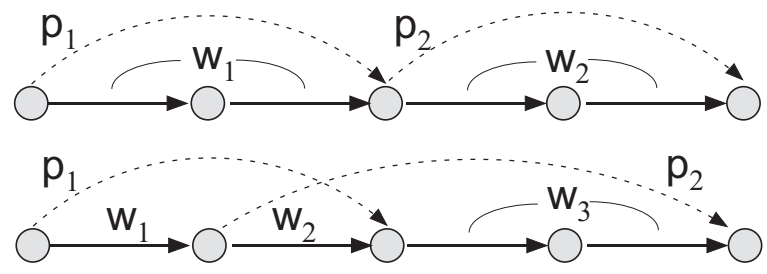

Fig. 1. (a) No-overlap and (b) overlap SP.

the affected WS is switched onto its BS, and the other WSs are unaware of the failure. In addition, in shared-segment protection (SSP), two BSs can share backup wavelength links as long as their WSs do not traverse the same link. Segment protection can be classified as overlap SP (o-SP), if the WS are allowed to overlap on some links, and no-overlap SP (no$\mathrm{SP}$ ), if WSs are strictly link-disjoint (see Fig. 1).

SP has a number of advantages compared to path protection: the end-to-end protection entity is a segment in segment protection as opposed to a path in path protection. Since a segment is typically shorter than a path in terms of hop count: i) SP is expected to have shorter protection-switching time, and $i i)$ the probability of two working segments sharing the same risk in segment protection is typically lower than the probability that two working paths in shared path protection share the same risk [9]. Note that if working segments/paths do not share the same risk group, then the respective backup segments/paths can share backup resources: as a result, segment protection can have better backup sharing and more routing flexibility compared to shared-path protection. In a general sense we can say that segment protection has more flexibility in routing compared to path protection since path protection is a special case of segment protection in which every path has exactly one segment.

Finally, segment protection is able to provide a higher availability degree with respect to the classical shared path protection: as a matter of fact, since the overlapped segments are protected by two backup segments (e.g., working segment $w_{2}$ in Fig. $1 \mathrm{~b}$ is protected by both $p_{1}$ and $p_{2}$ ), SP allows us to recover a larger number of double faults than shared path protection (e.g., a double fault affecting $w_{2}$ and $p_{2}$ can be still recovered along $p_{1}-w_{3}$ ).This qualitative conclusion will be confirmed by the findings of our analytical study in the rest of the paper.

\section{Availability Evaluation for Segment PROTECTION}

In order to verify if the availability requirements stated in Optical-SLAs are met, it is crucial to rely on appropriate analytical formulations to evaluate the availability $(A)$ target 


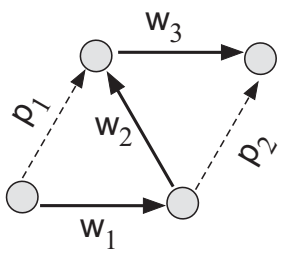

(a)

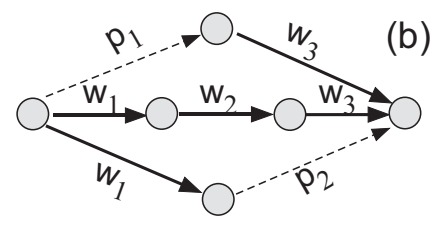

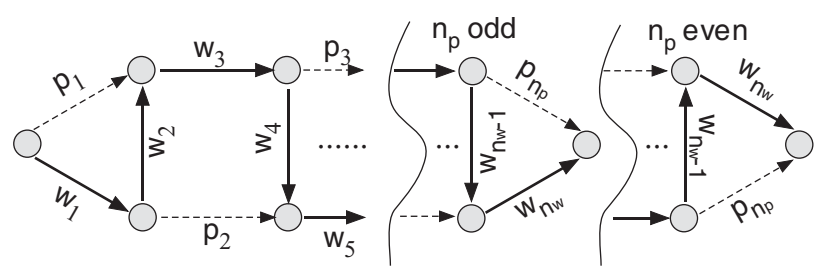

(c)

Fig. 2. (a) Original graph, (b) corresponding canonical graph, and (c) the generic case with $n_{p} \geq 2, n_{p}$ backup segments

of an SP-protected connection. Algebraic availability models for other well-known protection techniques have been already studied (e.g., in Ref. [5]). In this letter we consider availability models for Segment Protection; as far as we know, a complete algebraic availability analysis for Segment Protection has not been reported yet. In the literature, Ref. [6] presents an availability model only for the dedicated and no-overlapped case, which allows for a substantial simplification of the availability-evaluation problem. A very accurate formulation for reliability is provided in Ref. [7], but not for availability.

We start considering an exact evaluation of the availability in the Dedicated Segment Protection (DSP) case. To the best of our knowledge, this is the first time in literature the availability calculation for SP is solved exploiting the generating function (GF) method defined in Ref. [8], which allows us to solve exactly the overlapped case. Then, the same approach is applied to SSP, in association with an approximated methodology which provides accurate estimation of connection availability [5] in presence of shared backup resources.

\section{A. Dedicated Segment Protection}

The no-overlap DSP (no-DSP) case can be simply reduced to a series of dedicated protection schemes. If we define $A_{w_{i}}$ $\left(A_{p_{i}}\right)$ the availability of the $i$-th working (backup) segment, the availability $A_{i}$ of the $i$-th protected segment is $A_{i}=$ $A_{w_{i}}+A_{p_{i}}-A_{w_{i}} \cdot A_{p_{i}}$. Then, the availability of a $n o$-DSP connection formed by $n$ segments is given by $A=\prod_{i=1}^{n} A_{i}$. For example, in the case of Fig. 1a, the connection is divided in two segments $(n=2)$ and:

$$
\begin{aligned}
A= & A_{w_{1}} A_{w_{2}}+A_{p_{1}}\left(1-A_{w_{1}}\right) A_{w_{2}}+A_{p_{2}}\left(1-A_{w_{2}}\right) A_{w_{1}} \\
& +A_{p_{1}} A_{p_{2}}\left(1-A_{w_{1}}\right)\left(1-A_{w_{2}}\right)
\end{aligned}
$$

The overlap case introduces some additional complexity in this calculation. Some previous works solved the availability calculation recurring to the classical conditional decomposition approach [9]. Unfortunately, this approach fails in rigourously modeling the availability calculation for $o$-DSP, especially if applied to graphs with unidirectional links. E.g., let us consider the case in Fig. 1b, which has been re-drawn in Fig. 2a for sake of clarity: the conditional decomposition approach consists in decomposing the availability scheme in two separate cases, considering the "straddling link" $w_{2}$ (see Fig. 2a) either not available or perfectly available. Unfortunately this second hypothesis implies that the path $p_{1}-w_{2}-p_{2}$ could be used, because the series-parallel scheme does not take into account the directionality of link $w_{2}$.

So, a rigorous method to evaluate the availability in the graph of Fig. 2a consists in evaluating the GF [8]. As a first step, based on the original graph, a canonical graph composed by $m$ parallel branches, each representing a distinct path connecting the end-nodes, has to be built: e.g., in Fig. $2 \mathrm{~b}$ we have drawn the canonical graph of the structure in Fig. $2 a$ (n.b., now the path $p_{1}-w_{2}-p_{2}$ cannot be used). Once the canonical graph is built, the generating function to obtain the availability of a generic $o$-DSP configuration is defined as [8]:

$$
A=1-\prod_{i=1}^{m}\left(1-A_{T_{i}}\right)
$$

where i) $A_{T_{1}}, A_{T_{2}}, \ldots, A_{T_{m}}$ represent the availabilities of all the admissible paths $T_{1}, T_{2}, \ldots, T_{m}$ between the end nodes (in our example $m=3$ ) and ii) in the resolution of eq. 2 we must account "repeated events" only one time (e.g. $x y \cdot x z=x y z$, this operation is also referred to as "reduction rule"). This second operation is necessary to take into account the dependency between links in the canonical graph. For example, in the case of Fig. 2a we can write:

$$
\begin{aligned}
A= & 1-\left(1-A_{p_{1}} A_{w_{3}}\right)\left(1-A_{p_{2}} A_{w_{1}}\right)\left(1-A_{w_{1}} A_{w_{2}} A_{w_{3}}\right) \\
= & A_{w_{1}} A_{w_{2}} A_{w_{3}}+A_{p_{2}}\left(1-A_{w_{2}} A_{w_{3}}\right) A_{w_{1}}+A_{p_{1}} . \\
& \cdot\left(1-A_{w_{1}} A_{w_{2}}\right) A_{w_{3}}-A_{p_{1}} A_{p_{2}}\left(1-A_{w_{2}}\right) A_{w_{1}} A_{w_{3}}(3)
\end{aligned}
$$

For sake of clarity, let us consider the availability $A$ of the graph in Fig. 2a, for $A_{w_{1}}=0.9, A_{w_{2}}=0.8, A_{w_{3}}=$ 0.7, $A_{p_{1}}=0.25, A_{p_{2}}=0.36$ as in Ref. [9]. Our method returns $A=0.6622$, while $A=0.6863$ is obtained through conditional decomposition, with an overestimation (deriving from the fact that a non-feasible path is accounted) of about $3.6 \%$. Note that increasing the number of BSs such difference will further increase.

To extend the analysis to a general case with number of protection segments $n_{p} \geq 2$, let us consider, without loss of generality, the SP scheme in Fig. 2c: according to this scheme the number of working segments is $n_{w}=2 n_{p}-1$. Applying the GF methodology, a closed-form for the connection availability $A$ has been obtained (and can be checked) by Mathematica $\odot$ software and reported in Eq. 4, where $w_{i}$ is a working segment, $p_{i}$ is a protection segment, $W$ is the set of all working segments, $P$ is a specific subset of backup segments (specified in the following), $W_{p_{i}}$ is the set of working segments protected by the protection segment $p_{i}, W_{\cap}=W_{p_{j}} \cap W_{p_{j+1}}$ if $\forall j: p_{j} \wedge p_{j+1} \in P$ and $W_{\cup}=\bigcup W_{p_{j}}: p_{j} \in P$. Eq. 4 is composed by 4 terms: the first two terms refer to the cases of no- or a single failure, while the third and and fourth terms refer to the cases of two or more failures: in particular, the third term accounts only for the cases when each failed segment is protected by two segments (e.g. segment $w_{2}$ in Fig. 2c), while the fourth term accounts for all the remaining possible cases. $P$ contains all the backup segments that are activated in the third and 


$$
\begin{array}{r}
A=\prod_{i=1}^{n_{w}} A_{w_{i}}+\sum_{i=1}^{n_{p}} A_{p_{i}}\left(1-\prod_{w_{k} \subset W_{p_{i}}} A_{w_{k}}\right) \prod_{w_{m} \subset W-W_{p_{i}}} A_{w_{m}}-\sum_{i=1}^{n_{p}-1} A_{p_{i}} A_{p_{i+1}}\left(1+\sum_{l=i}^{n_{p}-2} \prod_{m=2}^{n_{p}-l} A_{p_{i+m}}\right)\left(1-\sum_{w_{k} \subset W_{\cap}} A_{w_{k}}\right) \\
\cdot \prod_{w_{m} \subset W-W_{n}} A_{w_{m}}+\sum_{i=1}^{n_{p}-1} A_{p_{i}} \sum_{n=i+2}^{n_{p}} A_{p_{n}}\left(1+\sum_{l=i}^{n_{p}-3} \prod_{m=3}^{n_{p}-l} A_{p_{i+m}}\right)\left(1-\sum_{p_{j} \subset P} \prod_{w_{k} \subset W_{p_{j}}} A_{w_{k}} \prod_{m \subset W-W \cup} A_{w_{m}}\right.
\end{array}
$$

fourth terms of Eq. 4 time by time by indices $i, n$ and $m^{1}$. Note that the total number of terms to be included in Eq. 4 is $1+\sum_{k=2}^{n_{p}}\left(\begin{array}{c}n_{p} \\ k\end{array}\right)$.

\section{B. Shared Segment Protection}

Let us start with the simplified case of shared-path protection: we know that a single backup channel can be used by different backup paths, as long as the associated working paths are node/link-disjoint working paths. Let us consider the case in which a backup link is shared by $N_{s}$ backup paths belonging to $N_{s}$ link-disjoint working paths $v_{i}$, each with availability $A_{v_{i}}$. According to the approximated approach shown in Ref. [5], the availability of such a structure can be evaluated as:

$$
A=A_{w}+A_{p}\left(1-A_{w}\right) \prod_{i=1}^{N_{s}} A_{v_{i}}
$$

where $N_{s}$ is the number of connections that share backup capacity along the backup path. Note that in Eq. 5, with respect to the dedicated case, we introduce $\prod_{i=1}^{N_{s}} A_{v_{i}}$, which represents a degradation term to take into account the availabilities of the working connections that share backup capacity with links along the backup path: increasing the number of paths that share backup capacity with the examined connection causes a decrease of the overall availability ${ }^{2}$.

The extension of this approach to the no-overlap segment protection case is obtained simply by multiplying the availability of each protected segments (evaluated as in Eq. 5).

For the overlap case, availability is obtained combining appropriately Eq. 4, which evaluates the availability of a $O$ SP connection in case of dedicated backup capacity, and Eq. 5, which includes the degradation terms due to the backup sharing. E.g., let us consider the case in Fig. 2a, composed by three working segments (i.e., $n_{w}=3$ ) and two protection segments (i.e., $n_{p}=2$ ). Let us assume the capacities of the first and second backup segment are shared by other $N_{1}$ and $N_{2}$ connections, respectively. The availabilities of the working paths that share backup capacities over the two

\footnotetext{
${ }^{1}$ Let us consider, as an example of the derivation of $P$, its application in the third term of Eq. 4; this term is composed of four elements: the product $A_{P}=A_{p_{i}} \cdot A_{p_{i+1}}$, two algebraic expressions in brackets and a final product; if we multiply $A_{P}$ by the two terms " 1 " appearing in the brackets, then the $W_{\cap}$ appearing in the last product will only contain $W_{p_{i}} \cap W_{p_{i+1}}$; if we do the same, but in the first expression in brackets we consider the first term of summation (i.e., $l=i$ ) instead of the term "1", then we have also the product of $A_{p_{i+2}} \ldots A_{p_{n_{p}-i}}$ and so $W_{\cap}$ appearing in the last product will contain $W_{p_{i}} \cap W_{p_{i+1}}$ and also $W_{p_{i+2}} \cap W_{p_{i+3}}$ and/or $W_{p_{i+3}} \cap W_{p_{i+4}}$ etc., if applicable.

${ }^{2}$ A more through discussion can be found in Ref. [5]. Other methods and techniques to evaluate the effect of backup sharing on availability have been proposed to in these last years [10]-[12], and can be applied to our framework for segment protection presented in this paper
}

backup segments are indicated by $A_{v_{i}^{\prime}}$ for the first backup segment and $A_{v_{i}^{\prime \prime}}$ for the second backup segment. Then, the availability is obtained combining Eq. 5 and Eq. 3 as in the following:

$$
\begin{aligned}
A= & A_{w_{1}} A_{w_{2}} A_{w_{3}}+A_{p_{1}}\left(1-A_{w_{1}} A_{w_{2}}\right) A_{w_{3}} \prod_{i=1}^{N_{1}} A_{v_{i}^{\prime}} \\
& +A_{p_{2}}\left(1-A_{w_{2}} A_{w_{3}}\right) A_{w_{1}} \prod_{i=1}^{N_{2}} A_{v_{i}^{\prime \prime}} \\
& -A_{p_{1}} A_{p_{2}}\left(1-A_{w_{2}}\right) A_{w_{1}} A_{w_{3}} \prod_{i=1}^{N_{1}} A_{v_{i}^{\prime}} \prod_{i=1}^{N_{2}} A_{v_{i}^{\prime \prime}}
\end{aligned}
$$

\section{NumericAl Results}

Let us analyze the SP availability through numerical examples. First, we analyze a single SP-protected connection, both in the dedicated and shared backup case, assuming that its working path $w$ has availability $A_{w}=0.9$ (i.e., each working segment has availability $A_{w_{i}}=A^{1 / n_{w}}$ ). Protection segments can be formed by either 1 or 3 links with availability $A_{p_{i}}=A^{\alpha / n_{w}}$ and $\alpha=1$ or $\alpha=3$, respectively. Second we apply our formulation in a network-wide scenario to compare the availability degree provided by some relevant proposal for routing of SSP connections.

\section{A. Dedicated Segment Protection}

For the dedicated case, Fig. 3 reports the unavailability $U=$ $1-A$ of $o$-DSP and no-DSP connections varying the number of backup segments. The value of $U$ decreases for increasing number of segments since, given the working path availability, for a higher value of segments the percentage of double faults that disconnect the connection becomes smaller. As a matter of fact, the number of double faults that disconnect the circuit grows less rapidly than the number of total double faults: e.g., in the scheme of Fig. 2c their ratio is given by $n_{p} /\left(\begin{array}{c}n_{p}+n_{w} \\ 2\end{array}\right)$.

$o$-DSP outperforms no-DSP especially for low numbers of backup segments. Again, this could be explained considering the percentage of recoverable double faults, which results to be higher in the overlap case thanks to the larger set of admissible paths induced by segment overlapping. Comparing results for different length of backup segments (1 vs. 3 links), shorter backup paths provide better performance, since they are characterized by a higher availability: unfortunately only highly connected networks allow for short backup segments.

\section{B. Shared Segment Protection}

With respect to the DSP case, in SSP the multiplicative terms accounting for the effect of sharing influence negatively 


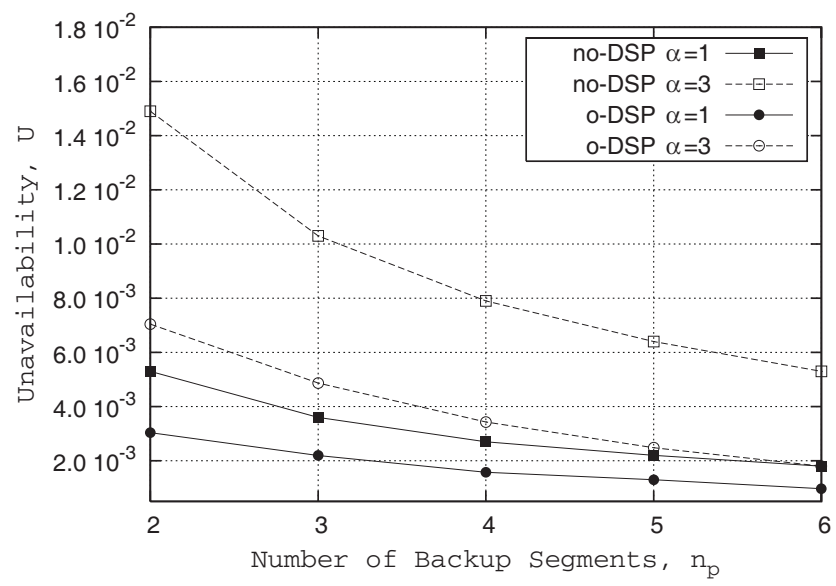

Fig. 3. Unavailability of overlap and non-overlap DSP connections for increasing number of backup segments.

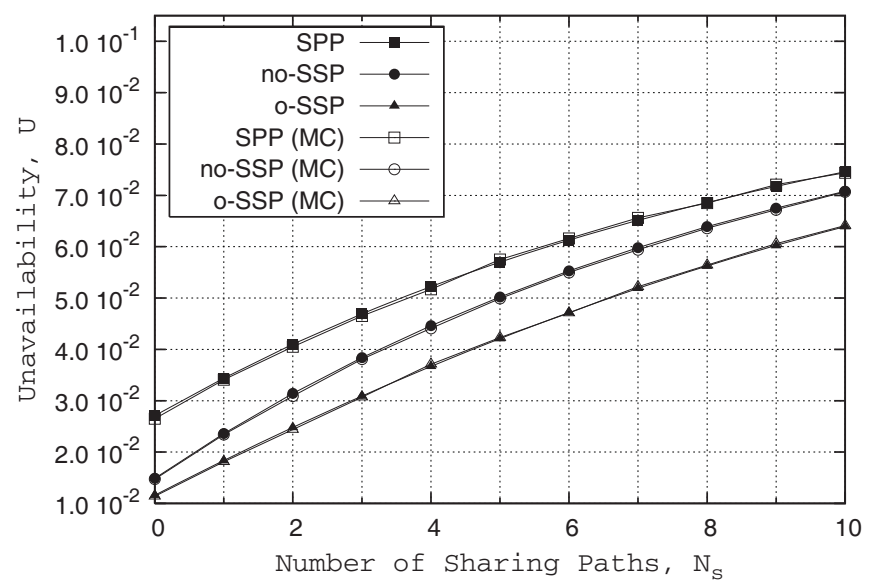

Fig. 4. Unavailability of overlap and non-overlap SSP connections varying the number of sharing paths.

the connection availability: these multiplicative terms are always smaller than unity and their values tend to decrease when the number of sharing connections grows. Fig. 4 reports the $U$ values for $o$-SSP and no-SSP connections, for increasing number of paths $N_{s}$ sharing their backup capacity, and considering protection segments formed by 3 links. Also values of $U$ for shared path protection (SPP) are reported for comparison. As anticipated, the unavailability increases with the number of sharing paths for any protection scheme. SSP configurations outperform SPP: this gain tends to decrease in presence of high sharing in the case of $n o-\mathrm{SSP}$, while it stays constant in the $o$-SSP case. Fixed the working path length and availability, the overlap configuration implies a larger number of backup segments and so a larger number of admissible paths and recoverable double faults.

For the previous results, we have applied Monte-Carlo (MC) simulations following the approach in Ref. [5] to verify the accuracy of our theoretical analysis, obtaining a very good convergence of analytical results and simulations. Comparisons are reported in Fig. 4 for SSP case (lines are almost overlapped), while in the DSP case analogous results are not reported for sake of brevity.

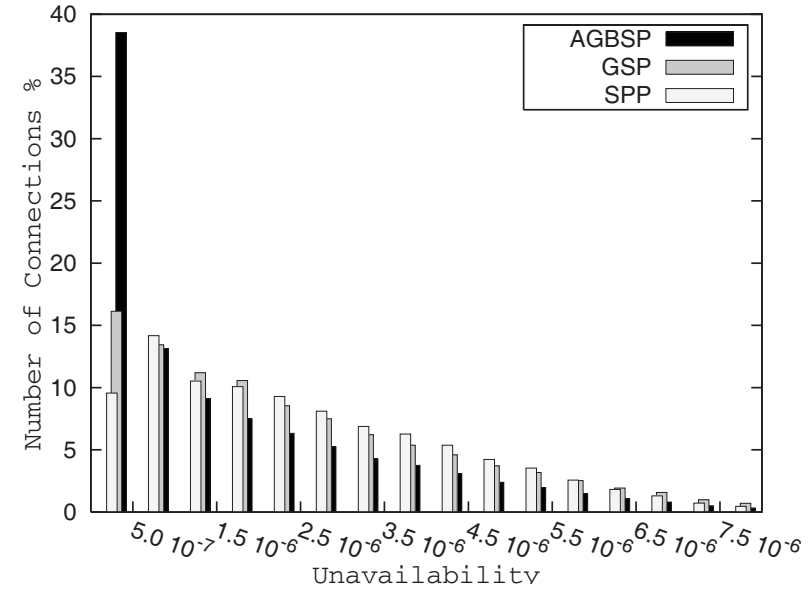

Fig. 5. Histogram of connection unavailability distribution for various SP algorithms in a dynamic environment.

\section{A network-wide analysis}

Using the previous equations, we can evaluate the availability degree provided by SP also in a network-wide scenario. We consider the same setting of the dynamic environment simulated in Ref. [1]: a US-backbone network topology equipped with 16 wavelengths per fiber is used, and 1,000,000 connections are offered with a Poissonian arrival rate of 100 connections per second and with unity negative exponential duration (leading to a load, normalized to network capacity, of around 0.4). Each link has availability $A_{l}=0.9999$.

Three algorithms are compared: shared path protection (SPP) and two options for shared segment protection, namely GSP from Ref. [1] and AGBSP from Ref. [4]. AGBSP, as compared to GSP, promotes a much better partitioning of the working path leading to a larger number of segments (see Ref. [4]). The histogram in Fig. 5 shows that SPP connections return on average an higher value of unavailability compared to GSP and AGBSP; furthermore, as anticipated in the previous subsection, an SSP algorithm which induces a more effective partitioning (as AGBSP) provides relevantly higher availability than algorithms that have less effective partitioning (GSP): in our scenario, by using AGBSP, a significant $38 \%$ of connections' unavailability falls in the class with lowest unavailability (i.e., $5 \cdot 10^{-7}$ ).

\section{CONCLusions}

A rigorous, yet efficient methodology is proposed which can be used to quantify the connection availability under segment protection. Effects of the number of segments and of external sharing paths on availability are shown and discussed.

\section{REFERENCES}

[1] C. Ou, S. Rai, and B. Mukherjee, "Extension of segment protection for bandwidth efficiency and differentiated quality of protection in optical/MPLS networks," Optical Switching Netw., pp. 19-33, Jan. 2005.

[2] Y. X. Dahai Xu and C. Qiao, "Novel algorithms for shared segment protection," IEEE J. Sel. Areas Commun., vol. 21, pp. 1320-1331, Nov. 2003.

[3] P.-H. Ho and H. T. Mouftah, "A novel survivable routing algorithm for segment shared protection in mesh WDM networks with partial wavelength conversion," IEEE J. Sel. Areas Commun., vol. 22, no. 8, pp. 1539-1548, Oct. 2004. 
[4] Y. Ouyang, Q. Zeng, and W. Wei, "Segment protection algorithm based on an auxiliary graph for wavelength-division multiplexing optical networks," OSA J. Optical Netw., vol. 5, no. 1, Jan. 2006.

[5] D. Arci, G. Maier, D. Petecchi, A. Pattavina, and M. Tornatore, "Availability models for protection techniques in WDM networks," in Proc. DRCN 2003, Banff, Canada, pp. 158-166, Oct. 2003.

[6] L. Guo, J. Cao, H. Yu, and L. Li, "Enhanced dynamic segment protection in WDM optical networks under reliability constraints," ETRI J., vol. 28, 2006.

[7] P. Ma, L. Zhou, and G. Mohan, "Dynamic routing of reliabilitydifferentiated connections in WDM optical networks," in Proc. IEEE Conf. Local Computer Netw. (LCN), 2005, pp. 190-199.

[8] C. Lee, "Analysis of switching networks," Bell Syst. Tech. J., no. 2, 1955.
[9] Q. She, X. Huang, and J. Jue, "Survivable routing for segment protection under multiple failures," in Proc. Optical Fiber Commun. Conf. 2007: Tech. Digest OFC/NFOEC, vol. JThA69, Mar. 2007.

[10] D. A. A. Mello, D. A. Schupke, and H. Waldman, "A matrix-based analytical approach to connection unavailability estimation in shared backup path protection," IEEE Commun. Lett., vol. 9, no. 9, pp. 844846, 2005.

[11] J. Zhang, K. Zhu, H. Zhang, N. Matloff, and B. Mukherjee, "Availability-aware provisioning strategies for differentiated protection services in wavelength-convertible WDM mesh networks," IEEE/ACM Trans. Networking, vol. 15, no. 5, pp. 1177-1190, 2007.

[12] L. Zhou, M. Held, and U.Sennhauser, "Connection availability analysis of shared backup path-protected mesh networks," IEEE/OSA J. Lightwave Technol., vol. 25, no. 5, pp. 1111-1119, May 2007. 